\title{
Linearly rigid metric spaces and the embedding problem
}

by

\author{
J. Melleray (Lyon), F. V. Petrov (St. Petersburg) \\ and A. M. Vershik (St. Petersburg)
}

Dedicated to the 95-th anniversary of L. V. Kantorovich

\begin{abstract}
We consider the problem of isometric embedding of metric spaces into Banach spaces, and introduce and study the remarkable class of so-called linearly rigid metric spaces: these are the spaces that admit a unique, up to isometry, linearly dense isometric embedding into a Banach space. The first nontrivial example of such a space was given by R. Holmes; he proved that the universal Urysohn space has this property. We give a criterion of linear rigidity of a metric space, which allows us to give a simple proof of the linear rigidity of the Urysohn space and some other metric spaces. Various properties of linearly rigid spaces and related spaces are considered.
\end{abstract}

\section{INTRODUCTION}

The goal of this paper is to describe the class of complete separable metric $(=$ Polish $)$ spaces $(X, \varrho)$ which have the following property: there is a unique (up to isometry) isometric embedding of $(X, \varrho)$ in a Banach space such that the affine span of the image of $X$ is dense (in which case we say that $X$ is linearly dense). We call such metric spaces linearly rigid.

The first nontrivial example of a linearly rigid space was Urysohn's universal space; this was proved by R. Holmes [9]. Recall that the Urysohn space is the unique (up to isometry) Polish space which is universal (in the class of all Polish spaces) and ultra-homogeneous (= any isometry between finite subspaces extends to an isometry of the whole space). It was

2000 Mathematics Subject Classification: 46B04, 51F10.

Key words and phrases: linear rigidity, isometric embedding, Kantorovich metric, Urysohn space.

Research of F. V. Petrov supported by grant NSh.4329.2006.1.

Research of A. M. Vershik supported by grants NSh.4329.2006.1 and RFBR.05-0100899 . 
discovered by P. S. Urysohn in his last paper [17], published after his tragic death. The criterion of linear rigidity which we give in this paper is a weakening of a well-known criterion of Urysohnness of a metric space, so the linear rigidity of the Urysohn space is an evident corollary of our theorem.

In this connection, in the first section we consider the general problem of isometric embedding of metric spaces into Banach spaces. There exist several functorial embeddings of an arbitrary metric space into a Banach space. The first one is the well-known Hausdorff-Kuratowski (HK) embedding into the Banach space of bounded continuous functions, $x \mapsto \varrho(x, \cdot)$. Our aim is to define an isometric embedding using suitable norms and seminorms compatible with the metric $\varrho$ on the free space over the metric space, or, in other words, seminorms in the space $V(X)$ of all finite affine combinations of elements of $X$. This method was used in [1], but it goes back to the idea of the 40s of the free group over a metric or topological space. The most important compatible norm is the KR-norm, which is based on the classical Kantorovich metric on the space of measures on the initial metric space; it was defined by L. V. Kantorovich in 1942 ([11]) in the framework of the Kantorovich-Monge transportation problem. The Kantorovich-Rubinstein (KR) norm, which is simply the extension of the Kantorovich metric to the space $V(X)$ (more exactly $V_{0}(X)$, see below), was defined in [12] for compact spaces.

A remarkable fact is that the KR-norm is the maximal norm compatible with the given metric, and a linearly rigid space is a space for which the KR-norm is the unique compatible norm.

The original definition of the KR-norm is direct, as the solution of a transportation problem; the main observation by Kantorovich was that the dual definition of the norm use the space conjugate to $V_{0}$, which is the space of Lipschitz functions (with Lipschitz norm) on the metric space. Thus the completion of $V_{0}$ under that norm is predual to the Banach space Lip of Lipschitz functions and is sometimes called the free Lipschitz space (see also $[5,10,21])$. It also worth mentioning that the geometry of the sphere in the KR-norm is nontrivial even for finite metric spaces and is related to the geometry of root polytopes of Lie algebras of series A, and other interesting combinatorial and geometrical questions. This geometry is directly connected with the problem of embedding finite metric spaces into a Banach space; the authors do not know if this question has ever been discussed systematically in full generality. Because of maximality of the KR-norm all other compatible norms and seminorms can be defined using some subspaces of the space of Lipschitz functions. We define a wider class of such norms; the main example of this class is what we call the double-point (dp) norm; it is used in the proof of the main theorem. 
In the second section we prove the main result, a criterion of linear rigidity in terms of distances. Namely, we prove that the characteristic property of those spaces is roughly speaking the following: any extremal (as the point of the unit sphere) Lipschitz function of norm 1 can be approximated by functions $\varrho(x, \cdot)+$ const. The characteristic property of the Urysohn space is stronger: one does not assume extremality of Lipschitz functions and there are some natural restrictions on the choice of the constant. We prove in particular that a metric space is linearly rigid if two norms: maximal $(K R)$ and double-point $(d p)$ coincide. We discuss some properties of linearly rigid spaces, for example, we prove that such a space must have infinite diameter; another property is that the unit sphere of it is completely degenerate in a sense.

In the third section we give several examples of linearly rigid spaces and first of all obtain Holmes's result about the linear rigidity of the Urysohn space using our criterion and compare it with the criterion of Urysohnness. The integral and rational (with distances more than 1) universal spaces are also linearly rigid. We also discuss some notions close to rigidity. One of them is the notion of almost universal space which has an approximation property that is stronger than for linearly rigid spaces, but weaker than for the Urysohn space; another one is the notion of weak linear rigidity of metric spaces which corresponds to the coincidence of HK- and KR-norms, which is weaker than linear rigidity.

We formulate several questions arising on the way. The geometry of those Banach spaces $E_{X}$ (and their unit spheres) which correspond to linearly rigid metric spaces $(X, \varrho)$ is very intriguing. The most important specific problem is to define axiomatically the Banach space $E_{\mathbb{U}}$ which corresponds to the Urysohn space $\mathbb{U}$, and can be called the Urysohn-Holmes-Kantorovich Banach space or briefly, the Urysohn Banach space. This is a very interesting universal Banach space with a huge group of linear isometries; it deserves to be considered from various points of view.

\section{ISOMETRIC EMBEDDINGS OF METRIC SPACES INTO BANACH SPACES}

1.1. Compatible norms and seminorms. Let $(X, \varrho)$ be a complete separable metric ( $=$ Polish) space $\left({ }^{1}\right)$. Consider the free vector space $V=$ $\mathbb{R}(X)$ over the space $X$, and the free affine space $V_{0}=\mathbb{R}_{0}(X)$ generated by the space $X$ (as a set) over the field of real numbers:

$\left({ }^{1}\right)$ The main definitions, assertions and proofs of the paper are also valid for nonseparable complete metric spaces. 


$$
\begin{aligned}
V(X) & =\mathbb{R}(X)=\left\{\sum a_{x} \delta_{x}: x \in X, a_{x} \in \mathbb{R}\right\} \\
& \supset V_{0}(X)=\mathbb{R}_{0}(X)=\left\{\sum a_{x} \delta_{x}: x \in X, a_{x} \in \mathbb{R}, \sum a_{x}=0\right\}
\end{aligned}
$$

(all sums are finite). The space $V_{0}(X)$ is a hyperplane in $V(X)$. We omit the mention of the space (and also of the metric, see below) if no ambiguity is possible; we will mostly consider only the space $V_{0}$. Another interpretation of the space $\mathbb{R}(X)$ (respectively, $\left.\mathbb{R}_{0}(X)\right)$ is that it is the space of real measures with finite support (respectively, the space of measures with finite support and total mass equal to zero: $\sum_{x} a_{x}=0$ ). Now we introduce the class of seminorms on $V_{0}$ compatible with the metric $\varrho$. For brevity, denote by $e_{x, y}=\delta_{x}-\delta_{y}$ the elementary signed measure corresponding to an ordered pair $(x, y)$.

Definition 1. We say that a seminorm $\|\cdot\|$ on $V_{0}(X)$ is compatible with the metric $\varrho$ on $X$ if $\left\|e_{x, y}\right\|=\varrho(x, y)$ for all $x, y \in X$.

Each compatible seminorm on $V_{0}$ can be extended to a seminorm on $V$ by setting $\left\|\delta_{x}\right\|=0$ for some $x \in X$, and vice versa, the restriction of a compatible seminorm on $V$ is a compatible seminorm on $V_{0}$; it is more convenient to consider compatible seminorms only on $V_{0}$.

The rays $\left\{c e_{x, y} \in V_{0}: c>0\right\}$ through elementary signed measures will be called fundamental rays (the set of fundamental rays does not depend on the metric). If the metric is fixed, then a compatible norm determines a unique vector of unit norm on each fundamental ray; let us call these vectors (elementary signed measures) fundamental vertices corresponding to the given metric. They are given by the formula $(x \neq y)$

$$
\frac{e_{x, y}}{\varrho(x, y)} \equiv \bar{e}_{x, y} \text {. }
$$

Thus the set of seminorms compatible with $\varrho$ is the set of seminorms for which the fundamental vertices corresponding to this metric are of norm one.

The following useful elementary lemma describes all possible metrics on a set $X$ in the geometrical terms of $V_{0}(X)$.

Lemma 1. Let $X$ be a set. Consider the linear space $V_{0}(X)$ and specify some points $c(x, y) \cdot e_{x, y}$ on the fundamental rays $\mathbb{R}_{+} \cdot e_{x, y}$, where $c(x, y)$ is a function defined for all pairs $(x, y), x \neq y$, positive, and symmetric: $c(x, y)=c(y, x)$. This set of points is the set of fundamental vertices of some metric on $X$ if and only if no point lies in the relative interior of the convex hull of a set consisting of finitely many other fundamental vertices and zero.

Proof. Define $\varrho(x, y)=c(x, y)^{-1}, x \neq y$, and $\varrho(x, x)=0$. Let us check that the triangle inequality for $\varrho$ is equivalent to the property of convex hulls 
mentioned in the lemma. First, if the triangle inequality does not hold, say $\varrho(c, a)>\varrho(c, b)+\varrho(b, a)$, then

$$
\bar{e}_{c, a}=\frac{\varrho(c, b)}{\varrho(c, a)} \cdot \bar{e}_{c, b}+\frac{\varrho(b, a)}{\varrho(c, a)} \cdot \bar{e}_{b, a} .
$$

The sum $\frac{\varrho(c, b)}{\varrho(c, a)}+\frac{\varrho(b, a)}{\varrho(c, a)}$ is less than 1 , hence $\bar{e}_{c, a}$ lies in the relative interior of the triangle with vertices $\bar{e}_{c, b}, \bar{e}_{b, a}$ and 0 .

Next, assume that $\varrho$ is a metric. We need to prove that the sum $\lambda=\sum \lambda_{i}$ of the coefficients in the representation

$$
\bar{e}_{x, y}=\sum_{i=1}^{n-1} \lambda_{i} \bar{e}_{x_{i}, y_{i}}, \quad \lambda_{i} \geq 0,
$$

is at least 1 . Integrate the function $\varrho(\cdot, y)$ with respect to the measures on both sides. By the triangle inequality,

$$
\int \varrho(\cdot, y) d \bar{e}_{x_{i}, y_{i}}=\frac{\varrho\left(x_{i}, y\right)-\varrho\left(y_{i}, y\right)}{\varrho\left(x_{i}, y_{i}\right)} \leq 1,
$$

so we get $1 \leq \sum \lambda_{i}$.

Let $\widehat{V}_{0}$ and $\widehat{V}$ be the quotients of $V_{0}$ and $V$ by the kernel $K=\{v:\|v\|=0\}$ of the seminorm, and let $\bar{V}$ and $\bar{V}_{0}$ be the norm completions of $\widehat{V}_{0}$ and $\widehat{V}$.

Proposition 1. Every compatible seminorm $\|\cdot\|$ on $V_{0}(X)$ defines an isometric embedding of $(X, \varrho)$ into the Banach space $(\bar{V},\|\cdot\|)$. Every isometric embedding of $(X, \varrho)$ into a Banach space $E$ corresponds to a compatible seminorm on $V_{0}(X)$.

Indeed, obviously, $(X, \varrho)$ has a canonical isometric embedding into $\bar{V}$, and conversely, it is easy to see that if there exists an isometric embedding of $(X, \varrho)$ into some Banach space $E$, then $V_{0}$ is also linearly embedded in $E$ and the restriction of the norm to $V_{0}$ defines a compatible seminorm (not a norm in general!) on $V_{0}$.

QUESTION 1. Let us call a metric space solid if each compatible seminorm is a norm. What metric spaces are solid? For example, what finite metric spaces are solid? A more concrete question: what is the minimal dimension of a Banach space into which a given finite metric space can be isometrically embedded?

A similar (but different) question is studied in [16].

Of course, it is enough to consider the case when the affine hull of the image of $X$ or the image of $V$ is dense in $E$. We will say that in this case the isometric embedding of $X$ into a Banach space $E$ is linearly dense. Thus, our problem is to characterize the metric spaces for which there is only one 
compatible norm or, equivalently, there is a unique, up to isometry, linearly dense embedding into a Banach space.

1.2. Examples, functorial embeddings. We will start with several important examples of isometric embeddings and compatible norms.

1.2.1. Hausdorff-Kuratowski embedding. The following is a well-known isometric embedding of an arbitrary metric space into a Banach space:

Definition 2. Define a map from the metric space $(X, \varrho)$ into the Banach space $\bar{C}(X)$ of all bounded continuous functions on $X$, endowed with the sup-norm, as follows:

$$
X \ni x \mapsto \varrho(x, \cdot), \quad V_{0} \ni e_{x, y} \mapsto \varrho(x, \cdot)-\varrho(y, \cdot) .
$$

We call it the Hausdorff-Kuratowski (HK) embedding.

It is evident that this embedding is an isometry. In general, it is not linearly dense, because the image of $V(X)$ consists of very special Lipschitz functions. It is difficult to describe exactly the closed subspace of $\bar{C}(X)$ that is the closed linear hull of the image under this embedding. At the same time, the corresponding compatible norm is given explicitly: for $v=\sum_{k} c_{k} \delta_{x_{k}}$ with $\sum_{k} c_{k}=0$,

$$
\left\|\sum_{k} c_{k} \delta_{x_{k}}\right\|=\sup _{z}\left|\sum_{k} c_{k} \varrho\left(z, x_{k}\right)\right| .
$$

1.2.2. Compatible norms which are defined by a class of Lipschitz functions. Let us give another example of a class of embeddings, which will play an important role. Choose a class $\mathcal{L} \subset \operatorname{Lip}(X)$ of Lipschitz functions which for any $x, y \in X$ contains a Lipschitz function $f_{x, y}(\cdot)$ such that $f_{x, y}(y)-f_{x, y}(x)=\varrho(x, y)$.

Definition 3. For every $v=\sum_{k} c_{k} \delta_{z_{k}} \in V_{0}$ set

$$
N_{\mathcal{L}}(v)=\sup _{f \in \mathcal{L}}\left|\sum c_{k} f\left(z_{k}\right)\right|
$$

Then $N_{\mathcal{L}}$ is a seminorm on $V_{0}$. We call it the $\mathcal{L}$-seminorm.

By definition we have $N_{\mathcal{L}}\left(e_{x, y}\right)=\varrho(x, y)$, so this seminorm is compatible with the metric $\varrho$. Note that any compatible seminorm can be obtained in this way by choosing a suitable subset $\mathcal{L}$ of Lipschitz functions.

Let us consider the following important specific example of seminorms (in fact, norms). Let $\mathcal{L}$ be the following class of 1-Lipschitz functions:

$$
\mathcal{L}=\left\{\phi_{x, y}(\cdot)=\frac{\varrho(y, \cdot)-\varrho(x, \cdot)}{2}: x, y \in X, x \neq y\right\} .
$$


The corresponding compatible norm is

$$
\|v\|=\sup _{x, y}\left|\sum_{k} c_{k} \phi_{x, y}\left(z_{k}\right)\right|,
$$

or in the notation for continuous measures $\mu$,

$$
\|\mu\|=\sup _{x, y}\left|\int \phi_{x, y}(z) d \mu(z)\right| \text {. }
$$

Definition 4. We will call this norm on $V_{0}(X)$ the double-point norm and denote it as $\|\mu\|_{\mathrm{dp}}$.

We will heavily use the double-point norm in the proof of the main result.

The functions $\phi_{x, y}$ in the above definition can be modified, for example, as follows: one may define

$$
\phi_{x, y}=\theta(x, y) \cdot \varrho(x, \cdot)-(1-\theta(x, y)) \cdot \varrho(y, \cdot)
$$

for any function $\theta: X^{2} \rightarrow(0,1)$ such that $\inf _{x, y} \theta(x, y)>0$ and $\sup _{x, y} \theta(x, y)$ $<1$.

1.2.3. Kantorovich embedding and maximal compatible norm. Now we consider the most important compatible norm: it arose as a consequence of the classical notion of Kantorovich (transport) metric ([11]) on the set of Borel measures on compact metric spaces, later called the KantorovichRubinstein norm [12].

The shortest way to define it is the following:

Definition 5. The Kantorovich-Rubinstein norm is the $N_{\mathcal{L}}$ norm where $N_{\mathcal{L}}$ is the set of all Lipschitz functions with Lipschitz norm 1; more directly, if $v=\sum_{k} c_{k} \delta_{z_{k}} \in V_{0}$ then

$$
\|v\|=\sup _{u}\left|\sum_{k} u\left(z_{k}\right) c_{k}\right|,
$$

where $u$ runs over all Lipschitz functions $u$ of norm 1:

$$
\|u\|=\sup _{x \neq y} \frac{|u(x)-u(y)|}{\varrho(x, y)}=1 .
$$

We can define this norm not only on $V_{0}$ but on the space of all Borel measures $\mu$ on the metric space $(X, \varrho)$ with compact support:

$$
\|\mu\|=\sup _{\|u\|=1}\left|\int_{X} u(z) d \mu(z)\right| .
$$

Immediately from the definition and from the remark above we have the following theorem.

Theorem 1. Let $(X, \varrho)$ be a Polish space. Then the KR-norm is the maximal compatible norm; we will denote it as $\|\cdot\|_{\max }$. 
In geometrical terms, this means that the unit ball in the KR-norm is the closed convex hull of the set of fundamental vertices $\bar{e}_{x, y}$ (see above); the unit ball with respect to every seminorm compatible with @ contains the unit ball in the KR-norm.

It is not difficult to prove this theorem directly. Recall that the initial definition of the Kantorovich-Rubinstein norm was different and the above theorem is the duality definition of the norm in comparison to the original; in that sense the definition above is the duality theorem due to L. Kantorovich ([11]). As already mentioned, the conjugate space to $V_{0}$ with the KR-norm is the Banach space of Lipschitz functions.

Denote the Banach space that is the completion of $V_{0}(X)$ with respect to the KR-norm by $E_{X, \varrho}$. Sometimes this space is called the "free Lipschitz space" [5]. It is easy to check that the correspondence

$$
(X, \varrho) \mapsto E_{X, \varrho}
$$

is a functor from the category of metric spaces (with Lipschitz maps as morphisms) to the category of Banach spaces (with bounded linear maps as morphisms).

Recall the initial definition:

Definition 6. Let $(X, \varrho)$ be a Polish space. For the Borel measure $\mu=$ $\mu_{+}-\mu_{-}$, where $\mu_{+}$and $\mu_{-}$are Borel probability measures, the KantorovichRubinstein norm is defined as

$$
\|\mu\|=\inf _{\psi} \int_{X} \int_{X} \varrho(x, y) d \psi(x, y)
$$

where $\psi$ runs over the set $\Psi=\Psi\left(\mu_{+}, \mu_{-}\right)$of all Borel probability measures on $X \times X$ with projections onto the first (resp. second) factor equal to $\mu_{+}$ (resp. $\mu_{-}$).

The equivalence of the two definitions of the KR-norm is precisely the duality theorem in linear programming.

1.2.4. Comments. 1. We have seen that the norm from Definition 6 defines a metric on the simplex of probability Borel measures: $\|\mu\| \equiv k_{\varrho}\left(\mu_{+}, \mu_{-}\right)$ (see Definition 7 below), and that is the metric initially defined by L. V. Kantorovich [11] $\left(^{2}\right)$. The same is true for all other compatible norms: each of them defines a metric on the affine simplex

$$
V_{0}^{+}(X)=\left\{v=\sum_{k} c_{k} \delta_{z_{k}} \in V_{0}(X): c_{k}>0, \sum_{k} c_{k}=1\right\}
$$

of probability measures by the formula $k_{\|\cdot\|}\left(v_{1}, v_{2}\right)=\left\|v_{1}-v_{2}\right\|, v_{1}, v_{2} \in V_{0}^{+}$.

$\left({ }^{2}\right)$ Many authors rediscovered this metric later; unfortunately, some of them did not mention the initial paper by L. V. Kantorovich (see the survey [19]). 
More generally, we can define the notion of compatible metric on $V_{0}^{+}(X)$ :

Definition 7. A metric $k_{\varrho}$ on the simplex $V_{0}^{+}(X)$ that is convex as a function on the affine set $V_{0}^{+}(X) \times V_{0}^{+}(X)$, and which has the property $k_{\varrho}\left(\delta_{x}, \delta_{y}\right)=\varrho(x, y)$, is called compatible with the metric space $(X, \varrho)$.

There are many compatible metrics which do not come from compatible norms as above. For example, the $L_{p}$-analogs, $p>1$, of the Kantorovich metric are not generated by compatible norms. The compatible metrics are very popular now in the theory of transportation problems (see [20]).

2. Our examples of compatible norms: Hausdorff-Kuratowski, doublepoint, maximal (Kantorovich-Rubinstein) norms etc., are functorial (with respect to isometries as morphisms) in the natural sense.

3 . In contrast to the existence of the maximal compatible norm there is no minimal compatible norm; moreover, it can happen that for a given compatible norm the infimum of the norms which are less than a given norm, is a seminorm, but not a norm.

4. The unit ball in the KR-norm for a finite metric space with all distances equal to 1 is nothing else than a generalization of root polytopes of Lie algebras of series A. More generally, the unit ball for a general metric could also be considered as a polytope in a Cartan subalgebra.

\section{MAIN THEOREM: CRITERION OF LINEAR RIGIDITY}

2.1. Criterion of linear rigidity. Let $(X, \varrho)$ be a metric space. We denote the Banach space of Lipschitz functions on $(X, \varrho)$ as $\operatorname{Lip}(X, \varrho)$ or $\operatorname{Lip}(X)$ and consider the quotient Banach space $\operatorname{PLip}(X)=\operatorname{Lip}(X) /\{$ const $\}$, both with the norm $\sup _{x \neq y}|u(x)-u(y)| / \varrho(x, y)$. The image of $u \in \operatorname{Lip}(X)$ under the projection $\pi: \operatorname{Lip}(X) \rightarrow \operatorname{PLip}(X)$ is denoted by $\widehat{u}$.

DEFINITION 8.

(1) The Lipschitz function of type $\phi_{x}(\cdot)=\varrho(x, \cdot)$ for some $x \in X$ is called a distance function.

(2) The Lipschitz function $u$ is called admissible if

$$
|u(x)-u(y)| \leq \varrho(x, y) \leq u(x)+u(y) .
$$

(3) Let $F \subset X$ and $u(\cdot)$ be a Lipschitz function on $F$ (equipped with the induced metric) of norm 1 . We say that $u$ is representable (respectively, $\varepsilon$-representable for some $\varepsilon>0)$ in $X$ if there exists a point $x \in X$ such that $u(z)=\varrho(x, z)$ (respectively $|u(z)-\varrho(x, z)|<\varepsilon)$ for all $z \in F$.

(4) We say that $u$ is additively representable (resp. additively $\varepsilon$-represent$a b l e)$ if there exists a constant $a$ in $\mathbb{R}$ such that the function $u(\cdot)-a$ is representable (resp. $\varepsilon$-representable). 
Now we formulate a criterion of linear rigidity of a metric space.

Recall that for a finite metric space $F$ a 1-Lipschitz function $f$ on $F$ is called extremal if it is an extreme point of the unit ball of $\operatorname{PLip}(F)$. In other words, whenever $f \pm g$ are both 1-Lipschitz, then $g$ must be a constant function.

THEOREM 2. The following assertions are equivalent:

(1) The complete metric space $(X, \varrho)$ is linearly rigid, that is, all norms on $V_{0}(X)$ compatible with $\varrho$ coincide.

(2) $\|\cdot\|_{\max }=\|\cdot\|_{\mathrm{dp}}$, i.e. the Kantorovich-Rubinstein norm and the double-point norm coincide.

(3) (criterion) For each finite subset $F \subset X$ (with the induced metric) and each $\varepsilon>0$, any extremal 1-Lipschitz function $u$ on $F$ is additively $\varepsilon$-representable.

(4) The weak* closure of the convex hull of the set of distance functions is the unit ball in $\operatorname{PLip}(X)\left({ }^{3}\right)$.

Recall that the weak* topology on $\operatorname{PLip}(X)$ is defined by duality between $V_{0}(X)$ and $\operatorname{PLip}(X)$.

Proof. (1) $\Rightarrow(2)$ is trivial.

$(2) \Rightarrow(3)$. Let $(X, \varrho)$ be a metric space for which the maximal and doublepoint norms coincide. Our goal is to prove that for any given finite $F \subset X$, any $\varepsilon>0$ and any extremal function $f$ on $F$ there exists a point $x \in X$ and a constant $a$ such that $\sup _{y \in F}|\varrho(x, y)-f(y)-a|<\varepsilon$.

If $F$ contains one or two elements, we may choose $x$ to be one of them, so let $F=\left\{x_{1}, \ldots, x_{n}\right\}, n \geq 3$.

Let us define a directed graph on $F$ as follows: draw an edge $x_{i} \rightarrow x_{j}$ if $f\left(x_{i}\right)-f\left(x_{j}\right)=\varrho\left(x_{i}, x_{j}\right)$. Note that this graph is connected. Indeed, otherwise for some disjoint nonempty sets $F_{1}, F_{2}$ such that $F=F_{1} \cup F_{2}$ there are no edges between $x_{i}$ and $x_{j}$ for any $x_{i} \in F_{1}, x_{j} \in F_{2}$. Then for small positive $\varepsilon$ the functions $f \pm \varepsilon \chi_{F_{1}}$ are also 1-Lipschitz, contrary to the extremality of $f$. (Here $\chi_{F_{1}}$ takes value 1 on $F_{1}$ and 0 on $F_{2}$ ). Define $\mu \in V_{0}(F)$ as

$$
\mu:=\sum e_{a, b}
$$

(here and until the end of the proof the summation is taken over all edges $a \rightarrow b$ of $F$ ). The linear functional $\nu \mapsto \int f d \nu$ attains its supremum on each function $e_{a, b}$ for any edge $a \rightarrow b$. Hence it also attains its maximum on the sum of these measures, i.e. on $\mu$. So

$$
\|\mu\|_{\max }=\int f d \mu=\sum \varrho(a, b) .
$$

$\left({ }^{3}\right)$ The last condition was suggested by the reviewer. 
Then also

$$
\|\mu\|_{\mathrm{dp}}=\sum \varrho(a, b)
$$

Hence for any $\varepsilon>0$ there exist $x, y \in X$ such that

$$
\left|\sum \varrho(a, b)-\sum\left(\phi_{x, y}(a)-\phi_{x, y}(b)\right)\right|<\varepsilon \text {. }
$$

We have

$$
\begin{aligned}
\varrho(a, b)-\left(\phi_{x, y}(a)-\phi_{x, y}(b)\right)= & (\varrho(a, b)-\varrho(x, a)+\varrho(x, b)) / 2 \\
& +(\varrho(a, b)+\varrho(y, a)-\varrho(y, b)) / 2 .
\end{aligned}
$$

Both summands are nonnegative and so both are less than $\varepsilon$ for any edge $a \rightarrow b$. This means that the function $g(\cdot)=\varrho(x, \cdot)-f(\cdot)$ is almost constant on $F$ (since $g(a)-g(b)$ is small for any edge and the graph is connected). So, $x$ satisfies the required conditions.

$(3) \Rightarrow(1)$. We prove that if (3) holds for any finite $F$, then for every signed measure $\mu \in V_{0}(F)$ and every norm $\|\cdot\|$ on $V_{0}(X)$ compatible with $\varrho$, one has $\|\mu\|=\|\mu\|_{K}$.

Recall that the unit ball of $\|\cdot\|_{K}$ is the closed convex hull of the points $\bar{e}_{a, b}$ : every finitely supported measure $\mu$ such that $\|\mu\|_{K}=1$ is a convex combination of some $\bar{e}_{a_{k}, b_{k}}$. Applying this to the measure $\mu /\|\mu\|_{K}$ we get

$$
\mu=\sum_{k=1}^{N} \alpha_{k} \bar{e}_{a_{k}, b_{k}}, \quad a_{k}, b_{k} \in F, \alpha_{k} \geq 0, \quad\|\mu\|_{K}=\sum \alpha_{k} .
$$

The points $\bar{e}_{a_{k}, b_{k}}$ lie on some face of the unit ball of the space $E_{F}$. We may assume without loss of generality that it is a face of codimension 1 . The corresponding supporting plane is determined by some linear functional of norm 1, i.e., some 1-Lipschitz function $f$ on $F$. Then for every $k, f\left(\bar{e}_{a_{k}, b_{k}}\right)=1$, that is, $f\left(a_{k}\right)-f\left(b_{k}\right)=\varrho\left(a_{k}, b_{k}\right)$. Hence $f$ is an extremal Lipschitz function on $F$.

First consider the case when all the $a_{k}$ are equal: $a_{k}=a$. By assumption, there is a point $c \in X$ such that $\varrho(c, a) \geq \varrho\left(c, b_{k}\right)+\varrho\left(a, b_{k}\right)-\varepsilon$. We have

$$
\begin{aligned}
\|\mu\| & =\left\|\sum \alpha_{k} \frac{\left(\delta_{a}-\delta_{c}\right)+\left(\delta_{c}-\delta_{b_{k}}\right)}{\varrho\left(a, b_{k}\right)}\right\| \\
& \geq \sum \alpha_{k} \frac{\varrho(a, c)}{\varrho\left(a, b_{k}\right)}-\sum \alpha_{k} \frac{\varrho\left(c, b_{k}\right)}{\varrho\left(a, b_{k}\right)} \geq \sum \alpha_{k}\left(1-\frac{\varepsilon}{\min _{k} \varrho\left(a, b_{k}\right)}\right) .
\end{aligned}
$$

Letting $\varepsilon \rightarrow 0$, we obtain

$$
\|\mu\|=\sum \alpha_{k}=\|\mu\|_{K} .
$$

Now consider the general case. Find a point $d$ such that $\varrho\left(d, a_{k}\right) \geq$ $\varrho\left(d, b_{k}\right)+\varrho\left(a_{k}, b_{k}\right)-\varepsilon$. Then we obtain 


$$
\begin{aligned}
\|\mu\| & =\left\|\sum \alpha_{k} \frac{\left(\delta_{a_{k}}-\delta_{d}\right)+\left(\delta_{d}-\delta_{b_{k}}\right)}{\varrho\left(a_{k}, b_{k}\right)}\right\| \\
& \geq\left\|\sum \alpha_{k} \frac{\delta_{a_{k}}-\delta_{d}}{\varrho\left(a_{k}, b_{k}\right)}\right\|-\sum \alpha_{k}\left\|\frac{\delta_{d}-\delta_{b_{k}}}{\varrho\left(a_{k}, b_{k}\right)}\right\| \\
& \geq \sum \alpha_{k} \frac{\varrho\left(a_{k}, d\right)}{\varrho\left(a_{k}, b_{k}\right)}-\sum \alpha_{k} \frac{\varrho\left(d, b_{k}\right)}{\varrho\left(a_{k}, b_{k}\right)} \geq \sum \alpha_{k}+o(1)
\end{aligned}
$$

(in the second inequality we use the case already considered). This completes the proof in the general case.

$(3) \Rightarrow(4)$ and $(4) \Rightarrow(3)$. It suffices to prove that any extremal Lipschitz function lies in the weak* closure of the set of distance functions (which consists of extremal Lipschitz functions itself). Take $u \in \operatorname{PLip}(X)$ which is extremal in the unit ball of $\operatorname{PLip}(X)$. It lies in the weak* closure of a set $L$ iff any weak* open neighborhood $W \ni u$ intersects $L$. But by the definition of the weak* topology such a neighborhood is defined by a finite subset of $X$, so we just get the criterion in (3).

We remark that it is possible to give a shorter proof of $(1) \Leftrightarrow(3)$ directly by using the Krein-Milman theorem; but the proof above is elementary, reduces the general case to the case of a finite metric space, and does not use infinite constructions. We will compare our criterion with the characteristic property of the Urysohn space in Section 3.

REMARK. We see that the coincidence of the double-point norm with the Kantorovich-Rubinstein norm implies the coincidence of all compatible norms. But instead of the double-point norm we can choose any norm which was defined in 1.3.1. The proof is essentially the same. So there are many ways to define a norm, compatible with the metric, whose coincidence with the Kantorovich the norm implies the linear rigidity.

\subsection{Properties of linearly rigid spaces}

2.2.1. Unboundedness of linearly rigid spaces. The following theorem shows that a linearly rigid space cannot have a finite diameter if it has more than two points.

THEOREM 3. A linearly rigid metric space $X$ containing more than two points is of infinite diameter and, in particular, is noncompact.

Proof. Suppose the contrary. Without loss of generality we assume that $X$ is complete. Fix $a \in X$, set $r_{a}=\sup _{x \in X} \varrho(a, x)$, and choose a sequence $\left(x_{n}\right)$ such that $\varrho\left(a, x_{n}\right) \geq r_{a}-1 / n$. Then pick a countable dense subset $\left\{y_{n}\right\}$ of $X$, and define a sequence $\left(z_{n}\right)$ by setting $z_{2 n}=x_{n}$ and $z_{2 n+1}=y_{n}$. Consider the points $\bar{e}_{a, z_{k}}, k=1, \ldots, N$. They lie on the same face of the unit ball of $E_{X_{N}}$, where $X_{N}=\left\{a, z_{1}, \ldots, z_{N}\right\}$. Applying Theorem 2 , we may 
find for each $N$ a point $c_{N}$ such that

$$
\varrho\left(a, c_{N}\right) \geq \varrho\left(a, z_{k}\right)+\varrho\left(z_{k}, c_{N}\right)-1 / N, \quad k \leq N .
$$

In particular, $\varrho\left(a, c_{N}\right) \geq \varrho\left(a, x_{k}\right)+\varrho\left(x_{k}, c_{N}\right)-1 / N, 2 k \leq N$. Hence $\varrho\left(a, c_{N}\right)$ $\rightarrow r_{a}$ and therefore $\varrho\left(x_{k}, c_{N}\right) \rightarrow 0$ as $k, N \rightarrow \infty$, so that the sequences $\left(x_{k}\right)$, $\left(c_{k}\right)$ are both Cauchy and have a common limit $a^{\prime}$. The point $a^{\prime}$ satisfies the equalities $\varrho(a, x)+\varrho\left(x, a^{\prime}\right)=\varrho\left(a, a^{\prime}\right)$ for all $x \in X$ (this is why we used the countable dense set $\left\{y_{n}\right\}$ in the definition of $\left.\left(z_{n}\right)\right)$.

Such a construction may be done for any point $a \in X$; note that for any $a, b \in X$ such that $a \neq b \neq a^{\prime}$ (such $a, b$ do exist if $X$ has more than two points) we have $2 \varrho\left(a, a^{\prime}\right)=\varrho(a, b)+\varrho\left(a, b^{\prime}\right)+\varrho\left(a^{\prime}, b\right)+\varrho\left(a^{\prime}, b^{\prime}\right)=2 \varrho\left(b, b^{\prime}\right)$, whence $\varrho\left(a, a^{\prime}\right) \equiv D<\infty$. It also follows that $\varrho(a, b)=\varrho\left(a^{\prime}, b^{\prime}\right)$.

Without loss of generality, $\varrho\left(a, b^{\prime}\right)=\varrho\left(b, a^{\prime}\right) \geq \varrho(a, b)=\varrho\left(a^{\prime}, b^{\prime}\right)=1$. Let $A=\left\{a, b, a^{\prime}, b^{\prime}\right\}$. Define a function $\varphi: A \rightarrow \mathbb{R}$ by the formulas $\varphi(a)=$ $\varphi\left(a^{\prime}\right)=1, \varphi(b)=\varphi\left(b^{\prime}\right)=0$.

Such a function is 1-Lipschitz on $\left\{a, b, a^{\prime}, b^{\prime}\right\}$; the corresponding face contains the points $\bar{e}_{a, b}, \bar{e}_{a^{\prime}, b^{\prime}}$. Hence there exists a point $c$ such that

$$
\varrho\left(c, a^{\prime}\right) \geq \varrho\left(c, b^{\prime}\right)+1 / 2, \quad \varrho(c, a) \geq \varrho(c, b)+1 / 2 .
$$

We have

$$
\varrho\left(a, a^{\prime}\right)=\varrho(a, c)+\varrho\left(c, a^{\prime}\right) \geq \varrho\left(c, b^{\prime}\right)+\varrho(c, b)+1=D+1 .
$$

This contradiction proves the theorem.

2.2.2. How to construct inductively a linearly rigid space. Let $(X, \varrho)$ be a linearly rigid metric space and $E_{X, \varrho}$ the corresponding Banach space. The properties of the unit sphere of $E_{X, \varrho}$ are very peculiar and can be used for the recursive construction of the metric space and the corresponding Banach space. We give a draft of the inductive construction; it is based on the following finite-dimensional

THEOREM 4 (Piercing theorem). Let $\left(Y_{1}, r_{1}\right)$ be an arbitrary finite metric space, $\varepsilon>0$, and $\Gamma$ be a face of the unit ball of the space $E_{Y_{1}, r_{1}}$ (e.g. $V_{0}\left(Y_{1}\right)$ with a compatible norm). Then the space $\left(Y_{1}, r_{1}\right)$ can be isometrically embedded into a finite metric space $\left(Y_{2}, r_{2}\right)$ so that there exists a face $\Delta$ of the unit ball of $E_{Y_{2}, r_{2}}$ containing $\Gamma$ and two vectors $\bar{e}_{z_{1}, z_{2}}$ and $\bar{e}_{u_{1}, u_{2}}$ such that the line segment connecting them intersects the face $\Delta$ at an interior point.

The proof is direct. Note that if an interior point of a face is of norm one, then all points of the face are also of norm one. Enumerating the sequences of faces of the root polytopes already constructed and "piercing" them by new line segments, we obtain a sequence of finite metric spaces for which all faces of all root polytopes are rigid; hence the completion of the constructed 
countable space will be linearly rigid. Note that such a degeneracy of the unit sphere is typical for universal constructions (cf. the Poulsen simplex [15]). We expect the geometry of the unit sphere of $E_{X, \varrho}$ for a linearly rigid metric space $(X, \varrho)$ to be unusual and interesting.

\section{EXAMPLES OF LINEARLY RIGID SPACES AND RELATED PROBLEMS}

Up to now we have not provided any example of a linearly rigid space. Trivial examples are one- and two-point spaces. R. Holmes [9] discovered that the universal Urysohn space has this property: the closed linear hulls of the images of all isometric embeddings of it in the universal space $C([0,1])$ are isometric Banach spaces, so in our terminology the Urysohn space is linearly rigid, and this was the first nontrivial example of such a space. We will deduce this result as well as other examples as easy consequences of our criterion.

\subsection{Criteria of Urysohnness and linear rigidity of the Urysohn}

space. In order to prove the linear rigidity of the Urysohn universal space we recall its characterization. The following criterion follows from the original paper of Urysohn [17] and subsequent papers [13, 18, 7].

Theorem 5. A Polish space $(X, \varrho)$ is isometric to the universal Urysohn space if and only if for every $\varepsilon>0$ and every finite subset $F \subset X$, each admissible Lipschitz function $u$ on $F$ is $\varepsilon$-representable in $X$ (see Definition 8.4).

From the point of view of functional analysis we can reformulate this condition as follows: the set of distance functions is weak* dense in the unit ball of $\operatorname{Lip}(X)$.

Theorem 6 (R. Holmes [9]). The Urysohn space $\mathbb{U}$ is linearly rigid.

Proof. It suffices to compare the assumptions of the criterion of universality above and the linear rigidity criterion from the previous section: the assumptions of the latter require additive $\varepsilon$-representability of extremal Lipschitz functions, while the universality criterion requires $\varepsilon$-representability of all positive Lipschitz functions.

In other words, according to item (4) of Theorem 2, the linear rigidity is equivalent to weak* density of the convex hull of the set of distance functions in the unit ball of $\operatorname{PLip}(X)$ while the universality is equivalent to the density of the set of distance functions in the unit ball of $\operatorname{Lip}(X)$, which is a much stronger condition. 
It is natural to call the Banach space $E_{\mathbb{U}}$ (completion of $V_{0}(\mathbb{U})$ with respect to the unique compatible norm) the "Urysohn Banach space" $\left({ }^{4}\right)$. The geometry of this space seems to be very interesting. First of all, $E_{\mathbb{U}}$ is universal as a Banach space, which means that each separable Banach space can be linearly isometrically embedded in it. As pointed out by Professor V. Pestov, this follows from a strong theorem of Godefroy and Kalton [6], which states that if some separable Banach space $F$ has an isometric embedding into a Banach space $B$, then it also has a linear isometric embedding into $B$.

However, $E_{\mathbb{U}, \varrho}$ is not homogeneous universal as a Banach space -it is known that there is no separable Banach space in which each linear isometry between any two finite-dimensional isometric linear subspaces can be extended to a global isometry of the space $([14,8,15])$. The Gurariu space ([8]) has an $\varepsilon$-version of this property.

QUESTION 2. Is the Urysohn Banach space $E_{\mathbb{U}}$ isometrically isomorphic to the Gurariu space?

\subsection{Further examples}

3.2.1. Rational discrete universal metric spaces are linearly rigid. Let us discuss other examples of linearly rigid universal spaces.

Let us consider the countable metric space denoted by $\mathbb{Q U} \geq 1$. It is a universal and ultra-homogeneous space in the class of countable metric spaces with rational distances not smaller than one. Such a space can be constructed in exactly the same way as the Urysohn space.

Theorem 7. The space $\mathbb{Q U}_{\geq 1}$ is linearly rigid.

Indeed, the assumptions of the criterion of linear rigidity are obviously satisfied.

This example, as well as the next one, is of interest because it is an example of a discrete countable linearly rigid space. Thus the corresponding Banach space $E_{\mathbb{Q U}}$ has a basis. It is not known whether the space $E_{\mathbb{U}}$ has a basis.

Definition 9. Let us call a metric space $(X, \varrho)$ almost universal if the set of distance functions is weak* dense in the unit ball of $\operatorname{PLip}(X)$.

This notion lies between universal Urysohn and linearly rigid spaces but does not coincide with any of them. It is easy to prove

Proposition 2. The space $\mathbb{Q U}_{\geq 1}$ is almost universal.

$\left({ }^{4}\right)$ Or Urysohn-Holmes-Kantorovich Banach space; in the case of coincidence of it with the Gurariŭ space (see Question 2 below) also add the name of Gurariu. 
At the same time the integral universal space is linearly rigid (see next subsection), but not almost universal.

Question 3. Describe all almost universal metric spaces.

3.2.2. The integral universal metric space is linearly rigid. The following example is of special interest also for another reason. Consider the space $\mathbb{Z} \mathbb{U}$, the universal and ultra-homogeneous space in the class of metric spaces with integer distances between points. Let us show that it is also linearly rigid. For this, let us check the condition of the criterion of linear rigidity. Fix a finite set $X_{n}$ in the space $X$ and an extremal ray $L=\{\lambda f\}, \lambda>0$, of the set of Lipschitz functions on $X_{n}$. Note that the differences of the coordinates of every vector from the ray $L$ are integers; hence on this ray there is a vector with integer coordinates, which is realized as the vector of distances between some point $x \in X$ and the points of the set $X_{n}$.

Let us introduce a graph structure on this countable space by assuming that pairs of points at distance one are neighbors. This graph has remarkable properties: it is universal but not homogeneous (as a graph), its group of isomorphisms coincides with the group of isometries of this space regarded as a metric space. As follows from $[3,4]$, there exists an isometry that acts transitively on this space.

QUESTION 4. Characterize this graph using its universality as a metric space.

3.3. Weakly linearly rigid spaces. We have proved that the coincidence of KR- and dp-norms leads to linear rigidity. Now let us compare another two compatible norms: the KR-norm and HK-norm.

Definition 10. A metric space for which the maximal (or KR-) norm and HK-norm coincide is called a weakly linearly rigid space (WLR-space).

If $(X, \varrho)$ is linearly rigid, then all compatible norms must coincide with the maximal norm of $\sum c_{k} \delta_{x_{k}}$, which is

$$
\left\|\sum c_{k} \delta_{x_{k}}\right\|_{\mathrm{HK}}=\left\|\sum c_{k} \delta_{x_{k}}\right\|_{\mathrm{KR}}=\sup _{F \in \operatorname{Lip}_{1}(X)} \sum c_{k} F\left(x_{k}\right),
$$

where the supremum is taken over all 1-Lipschitz functions on $X$ (or, equivalently, on the set of $\left\{x_{k}\right\}$ ). The equality above means that every extremal Lipschitz function almost coincides with the function $\pm \varrho(x, \cdot)$ for some $x \in X$. The criterion of linear rigidity says that any extremal Lipschitz function on $X$ almost coincides with a function $\varrho(x, \cdot)$. The only difference is the absence of \pm . Recall that if we take the supremum not over the functions $\pm \varrho(x, \cdot)$, but over the functions $\frac{1}{2}(\varrho(x, \cdot)-\varrho(y, \cdot))$, then the coincidence of the corresponding norm with the maximal (KR) norm implies linear rigidity. But this difference is quite essential. There are some spaces which are 
weakly linearly rigid but not linearly rigid. We do not have a sufficiently complete description of such spaces. So only some examples follow.

1. Any metric space on three points is a WLR-space (but it is never linearly rigid, see Theorem 3 ).

2. Let us define a family of four-point WLR-spaces. Let the points be $A$, $B, C, D$, let $a, b, c$ be arbitrary positive numbers, and define $\varrho(D, A)=a$, $\varrho(D, B)=b, \varrho(D, c)=c, \varrho(A, B)=a+b, \varrho(B, C)=b+c, \varrho(A, C)=a+c$. It is easy to see that any extremal Lipschitz function is $\pm \varrho(X, \cdot)$ for some $X \in\{A, B, C, D\}$.

Question 5. Does there exist a finite WLR-space with more than four points?

It seems likely that there are no such spaces, at least with sufficiently many points.

3. There is an infinite WLR-space which is not linearly rigid. Consider the Urysohn space $\mathbb{U}$ and fix a point $a \in \mathbb{U}$. Add a point $a^{\prime}$ to $\mathbb{U}$ and define $\varrho\left(a^{\prime}, x\right)=\varrho(a, x)+1$ for any $x \in X$ (in particular, $\varrho\left(a, a^{\prime}\right)=1$ ). Then $U^{\prime}=\mathbb{U} \cup\left\{a^{\prime}\right\}$ is WLR, but not linearly rigid.

\subsection{Extremality and the properties of the Banach spaces $E_{X, \varrho}$.} The set of all possible distance matrices (semimetrics) is a convex weakly (i.e. in pointwise topology) closed cone (see [18]). If the distance matrix of a Polish space $X$, which corresponds to some dense sequence in $X$, belongs to an extremal ray of this cone, we say that $X$ is extremal. This property does not depend on the choice of the dense sequence, so the definition is correct. This notion is interesting even for finite metric spaces (see [2]).

Question 6. Describe extremal finite metric spaces with $n$ points, estimate the exact number of such spaces or give its asymptotics in $n$.

Note that the universal real Urysohn space $\mathbb{U}$ is an extremal metric space $([18])$. It follows from the genericity of $\mathbb{U}$ that the distance matrices of everywhere dense systems of points of extremal metric spaces form an everywhere dense $G_{\delta}$-set in the space of distance matrices. The integer space $\mathbb{Z} U$ is also extremal; the extremality of both spaces follows from a result of Avis [2], which states that every finite metric space with commensurable distances (i.e. such that the ratio of any two distances is rational) can be embedded into a finite extremal metric space, and hence the assumptions of the criterion of linear rigidity are satisfied. Using the criterion of linear rigidity given above, and the procedure of the previous section, one can build an example of a nonextremal linearly rigid metric space.

Acknowledgments. The authors are grateful to V. Pestov for useful discussions and to the reviewers for valuable suggestions. 


\section{References}

[1] R. Arens and J. Eells, On embedding uniform and topological spaces, Pacific J. Math. 6 (1956), 397-403.

[2] D. Avis, On the extreme rays of the metric cone, Canad. J. Math. 32 (1980), 126-144.

[3] P. Cameron, Homogeneous Cayley objects, Eur. J. Combin. 21 (2000), 745-760.

[4] P. Cameron and A. M. Vershik, Some isometry groups of the Urysohn space, Ann. Pure Appl. Logic 143 (2006), 70-78.

[5] V. Dutrieux and V. Ferenci, The Lipschitz free Banach space of $C(K)$-spaces, Proc. Amer. Math. Soc. 134 (2006), 1039-1044.

[6] G. Godefroy and N. Kalton, Lipschitz-free Banach spaces, Studia Math. 159 (2003), 121-141.

[7] M. Gromov, Metric Structures for Riemannian and Non-Riemannian Spaces, Birkhäuser Boston, Boston, 1999.

[8] V. I. Gurariü, Spaces of universal placement, isotropic spaces and a problem of Mazur on rotations of Banach spaces, Sibirsk. Mat. Zh. 7 (1966), 1002-1013 (in Russian).

[9] R. Holmes, The universal separable metric space of Urysohn and isometric embeddings thereof in Banach spaces, Fund. Math. 140 (1992), 199-223.

[10] N. J. Kalton, Spaces of Lipschitz and Hölder functions and their applications, Collect. Math. 55 (2004), 171-217.

[11] L. V. Kantorovich, On the translocation of masses, Dokl. Akad. Nauk SSSR 37 (1942), 227-229 (in Russian).

[12] L. V. Kantorovich and G. Sh. Rubinshtel̆n, On a space of totally additive functions, Vestnik Leningrad. Univ. 13 (1958), no. 7, 52-59 (in Russian).

[13] M. Katětov, On universal metric spaces, in: General Topology and Its Relations to Modern Analysis and Algebra, VI (Prague, 1986), Heldermann, Berlin, 1988, 323-330.

[14] J. Lindenstrauss, Extension of compact operators, Mem. Amer. Math. Soc. 48 (1964).

[15] W. Lusky, The Gurarij spaces are unique, Arch. Math. (Basel) 27 (1976), 627-635.

[16] S. A. Shkarin, Isometric embedding of finite ultrametric spaces in Banach spaces, Topology Appl. 142 (2004), 13-17.

[17] P. S. Urysohn, Sur un espace métrique universel, Bull. Sci. Math. 51 (1927), 1-28.

[18] A. M. Vershik, Random metric spaces and universality, Russian Math. Surveys 59 (2004), 259-295.

[19] - Kantorovich metric: initial history and little-known applications, J. Math. Sci. 133 (2006), 1410-1417.

[20] C. Villani, Topics in Optimal Transportation, Amer. Math. Soc., Providence, RI, 2003.

[21] N. Weaver, Lipschitz Algebras, World Sci., Singapore, 1999.

UFR de Mathématiques

Université Claude Bernard Lyon 1

43 boulevard du 11 novembre 1918

69622 Villeurbanne Cedex, France

E-mail: melleray@matgh.univ-lyon1.fr
Steklov Mathematical Institute at St. Petersburg

Fontanka 27

St. Petersburg 191023, Russia

E-mail: fedorpetrov@mail.ru vershik@pdmi.ras.ru

Received 13 November 2006;

in revised form 7 March 2008 